\title{
Interleukin-32 $\theta$ inhibits tumor-promoting effects of macrophage-secreted CCL18 in breast cancer
}

\author{
Thu-Huyen Pham ${ }^{1 \dagger}$, Yesol Bak ${ }^{1 \dagger}$, Taeho Kwon², Sae-Bom Kwon', Jae-Wook Oh³ , Jong-Hyung Park ${ }^{4}$, \\ Yang-Kyu Choi ${ }^{4}$, Jin Tae Hong ${ }^{5 *}$ and Do-Young Yoon ${ }^{1 *}$ (D)
}

\begin{abstract}
Background: Tumor-associated macrophages can promote breast cancer metastasis by secreting cytokines and growth factors. Interleukin (IL)-320, a newly identified IL-32 isoform, was previously shown to down-regulate various proinflammatory factors of macrophages. Here, we report the presence of IL-32 $\theta$ in breast cancer tissues and evaluate its effects on macrophage-regulated breast cancer metastasis.

Methods: RT-qPCR was used to analyze the mRNA expression of IL-320, Chemokine (C-C motif) ligand 18 (CCL18) in breast cancer tissues. In vitro cell-based experiments using IL-320-expressing MDA-MB-231 cells were conducted to examine the effects of IL-32 $\theta$ on metastasis and its molecular signaling. In vivo xenograft, immunohistochemistry, and optical imaging models were generated to support in vitro and clinical findings.

Results: The clinical data displayed opposite expression patterns of CCL18 and IL-32 $\theta$ mRNA in macrophageinfiltrated breast tumor tissues compared with those in the other tissues tested. In MDA-MB-231 cells, IL-32 $\theta$ overexpression attenuated migration, invasion, tumor-promoting factors, and increased epithelial markers levels upon treatment with conditioned media from THP-1-derived macrophages. Additionally, IL-32 $\theta$ expression in a xenograft model led to a remarkable decrease in tumor size and macrophage-stimulated tumor promotion. This inhibition was mediated through a direct interaction with protein kinase $C-\delta(P K C \delta)$, subsequently eliminating the downstream factors STAT3 and NF-KB. Blocking CCL18 during co-culture of macrophages and breast cancer cells reduced the levels of breast cancer progression-related factors and PKC $\delta$ downstream signaling suggesting CCL18 as the main macrophage-secreted factors triggering the signaling pathway inhibited by IL-320.
\end{abstract}

Conclusions: Our findings demonstrate a novel role of IL-32 $\theta$ as an intracellular modulator to suppress macrophage-promoted breast cancer progression by targeting CCL18-dependent signaling.

Keywords: IL-320, Macrophage, Breast cancer metastasis, CCL18, PKCס, STAT3

\section{Background}

Breast cancer is the most common cancer in females worldwide, and is also the leading cause of cancerrelated death in the majority of countries [1]. Tumor progression is the process by which tumor cells acquire

\footnotetext{
*Correspondence: jinthong@chungbuk.ac.kr; ydy4218@konkuk.ac.kr

${ }^{\dagger}$ Thu-Huyen Pham and Yesol Bak are contributed equally to this work.

${ }^{5}$ College of Pharmacy and Medical Research Center, Chungbuk National University, Osongsaengmyeong 1-ro, Osong-eup, Heungdeok-gu, Cheongju, Chungbuk 28160, Republic of Korea

${ }^{1}$ Department of Bioscience and Biotechnology, Konkuk University, 120 Neungdong-ro, Jayang-dong, Gwangjin-gu, Seoul 05029, Republic of Korea Full list of author information is available at the end of the article
}

more aggressive and malignant characteristics, allowing them to invade microenvironments and subsequently migrate to distant organs $[2,3]$. In this process, epithelial-mesenchymal transition (EMT) is one of the key events that allows tumor cells to switch to mesenchymal phenotypes to facilitate their migration, invasion, and metastasis [4]. This tumor metastasis and acquired resistance to tumor therapy is a result of the interaction between cancer cells and the tumor microenvironment, leading to the secretion of various factors that target cancer cells and manipulate their promotion [5-7].

(c) The Author(s). 2019 Open Access This article is distributed under the terms of the Creative Commons Attribution 4.0 International License (http://creativecommons.org/licenses/by/4.0/), which permits unrestricted use, distribution, and 
Therefore, inhibition of these interactions can serve as a therapeutic approach in cancer.

Macrophages are the most abundant immune cells in the tumor microenvironment, which can occupy up to $50 \%$ of the entire tumor mass [8], and have been associated with poor outcomes in various carcinomas [9]. Macrophages can be classified into M1 and M2 macrophages, which polarize into the respective forms in response to an environmental change. M2 macrophages facilitate angiogenesis, tissue remodeling [10], and promote breast cancer progression by secreting angiogenic factors and breast tumor mitogens [11]. Tumorassociated macrophages (TAMs) are a type of M2 macrophages, and breast cancer TAMs display an alternative phenotype that promotes tumor invasion and metastasis [12]. Further, cancer cells can educate macrophages to enhance tumor development and metastasis [6]. Numerous studies have determined the relationship between breast cancer and macrophages, and cancer therapies targeting both breast cancer cells and macrophages are of great interest given their potential in the clinical setting.

Interleukin (IL)-32 was first reported as natural killer transcript 4 located on human chromosome 16p13.3 [13]. IL-32 has various isoforms due to alternative splicing [14], although the role of each isoform in disease remains controversial [15]. Among the IL-32 isoforms, our group discovered both IL-32 $\theta$ and IL-32 small fragment [14] and reported the functions of IL-32 $\theta$ in inflammation and cancer [16-18]. In the present study, we aimed to investigate the role of IL-32 $\theta$ in the breast cancer microenvironment and to determine whether IL-32 $\theta$ could suppress macrophage-induced breast cancer progression, and to explore the underlying molecular mechanisms.

\section{Materials and methods mRNA extraction from breast cancer tissue}

The biospecimens including breast tissues $(n=90)$ and sera $(n=55)$, and the characteristic information of breast cancer patients used in this study were provided by the Biobank of Chonnam National University Hwasun Hospital (Hwasun-gun, Korea) and Korea University Guro Hospital (Seoul, Korea). RNA was extracted from frozen tissues using a homogenizer and TRI Reagent ${ }^{\circ}$ (Ambion, Austin, TX), and then CDNA was synthesized using the M-MuLV reverse transcriptase (New England Biolabs, Beverly, MA) according to manufacturer's instructions.

\section{Cell culture and treatment}

The MDA-MB-231 cell line (ATCC ${ }^{\bullet}$ HTB-26 $^{\text {tm }}$, Manassas, VA) was cultured in DMEM (Hyclone Laboratories, Logan, UT). The human monocytic cell line THP-1 (Korean Cell Line Bank, Seoul, Korea, KCLB-40202) was cultured in RPMI-1640 (HyClone). Both mediums were supplemented with $10 \%$ heat-inactivated fetal bovine serum (MilliporeSigma, Burlington, MA), 100 units $/ \mathrm{mL}$ penicillin, and $100 \mu \mathrm{g} / \mathrm{mL}$ streptomycin at $37^{\circ} \mathrm{C} / 5 \% \mathrm{CO}_{2}$. To generate the conditioned media (CM), THP-1 cells were stimulated with $100 \mathrm{nM}$ phorbol ester (PMA) (MilliporeSigma) for $48 \mathrm{~h}$, the non-attached cells were washed with phosphate buffered saline (PBS) followed by addition of fresh culture media, and these cells were then incubated for another $24 \mathrm{~h}$. The CM was collected and centrifuged to remove the remaining cells.

\section{Generation of the IL-32 $\theta$-overexpressing cell line}

We transfected MDA-MB-231 cells with the pcDNA3.1 $(+)-6 \times$ Myc-IL-32 $\theta$ vector or pcDNA3.1 (+)- $6 \times$ Mycempty vector, as described previously [19] and refer as MDA-MB-231-IL-32 $\theta$ and MDA-MB-231-EV cells, respectively. In brief, the cells were seeded into 6-well plates $\left(1 \times 10^{5}\right.$ cells/well $)$ and transfected with $3 \mu \mathrm{g}$ of vector using Lipofectamine 2000 (Invitrogen, Carlsbad, CA). Afterwards, the cells were selected using medium containing $700 \mu \mathrm{g} / \mathrm{ml} \mathrm{G}-418$ (Duchefa Biochemie BV, Haarlem, The Netherlands) for two weeks. G-418resistant colonies were then pooled and expanded.

\section{Migration and invasion assays}

For the migration assay, cells $\left(5 \times 10^{5}\right.$ cells $\left./ \mathrm{mL}\right)$ were seeded onto the upper chambers of 24-well transwell plates (Corning Inc., Corning, NY) in serum-free DMEM. For the invasion assay, each transwell chamber was coated with $30 \mu \mathrm{l}$ of Matrigel (Corning) before breast cancer cells were added to the top chamber. The lower chamber contained $500 \mu \mathrm{l}$ macrophage-derived CM. Cells migrated or invaded for $24 \mathrm{~h}$ at $37^{\circ} \mathrm{C} / 5 \%$ $\mathrm{CO}_{2}$, and non-migrated or non-invaded cells were removed from the chamber interior by a cotton swab. Attached cells to the lower surface of the chamber were stained using Diff-Quick Kit (Sysmex, Kobe, Japan). Migrated or invaded cells were quantitated by dissolving stained cells in $100 \mu \mathrm{l}$ of $10 \%$ acetic acid and then the mixture was transferred to a 96-well plate for colorimetric reading at $620 \mathrm{~nm}$.

\section{Quantitative reverse transcription PCR (RT-qPCR)}

The mRNA expression levels in breast cancer cells were detected by RT-PCR for IL-32 $\theta$ and RT-qPCR for other target genes. Total RNA was isolated using Easy-BLUE (iNtRON Biotechnology, SungNam, Korea), then reverse transcription was performed. qPCR was conducted using SensiFAST $^{\mathrm{m}}$ SYBR NO-ROX Kit (BIOLINE, London, UK). Samples were analyzed using the primer sets listed in Additional file 1: Table S1. Transcript levels were quantitated using the $-\Delta \mathrm{Ct}$ method $(\mathrm{Ct}=$ fluorescence threshold value; $-\Delta \mathrm{Ct}=\mathrm{Ct}$ GAPDH $-\mathrm{Ct}$ target gene). 
Enzyme-linked immunosorbent assay (ELISA)

Cells were cultured in the absence or presence of CM for $24 \mathrm{~h}$, and then the culture media were replaced by fresh media for another $24 \mathrm{~h}$. The cell culture supernatants were collected and analyzed using ELISA kits (R\&D Systems, Minneapolis, MN) for human IL-1 $\beta$, CCL5, CCL18, GM-CSF according to manufacturer's instructions.

\section{Immunoblotting and immunoprecipitation}

For nuclear and cytoplasmic fractionation, cells were collected and fractionated using the NE-PER kit (Thermo Fisher Scientific, Waltham, MA) according to manufacturer's instructions. For immunoprecipitation, cell lysates were mixed with specific antibodies and then pulled down by protein G-agarose beads. Samples were subjected to $10 \%$ SDS-PAGE before being transferred to PVDF membranes (MilliporeSigma). The membranes were blocked with $5 \%$ skim milk dissolved in Trisbuffered saline containing $0.05 \%$ Tween-20 followed by primary antibody incubation at $4{ }^{\circ} \mathrm{C}$ overnight. After washing, horseradish peroxidase-conjugated IgG antibodies were added, and the membranes allowed to incubate for $1 \mathrm{~h}$. Western blot was visualized using a chemiluminescence detection kit (Advanstar, Cleveland, Ohio) and detected by EZ-capture MG protein imaging system (ATTO, Tokyo, Japan). Specific antibodies used include those against Myc-tag, Flag-tag and phosphotyrosine-STAT3 (MilliporeSigma); I $\mathrm{B} \alpha, \mathrm{p}-\mathrm{I} \kappa \mathrm{B} \alpha$, p65, p50, PARP, and E-cadherin (Cell Signaling Technology, Danvers, MA); STAT3, COX-2, GAPDH (Santa Cruz Biotechnology, Dallas, TX); and anti-CCL18 neutralizing antibody (Abcam, Cambridge, MA). The monoclonal antibody KU-32-52 to detect IL-32 was prepared as previously described [20]. The raw data of western blot results can be seen in Additional file 2 .

\section{Gelatin zymography}

Cells $\left(3 \times 10^{5}\right.$ cells/well $)$ were seeded in a 6 -well plate, cultured overnight, and then treated with or without CM for 24h. MMP-9 activity in the supernatant was assayed as previously described [21]. Gel staining was conducted with InstantBlue $^{\mathrm{Tm}}$ (MilliporeSigma) for 30 min in the dark. Areas of gelatinolytic degradation appeared as transparent bands on the blue background.

\section{Immunofluorescence}

Cells were seeded on coverslips and incubated overnight. The attached cells were fixed, and permeabilized with cold acetone before blocking with $0.1 \%$ bovine serum albumin in PBS at room temperature (RT). Primary antibodies were added (1:100) to the coverslip incubating at $4{ }^{\circ} \mathrm{C}$ overnight. After washing with PBS, the coverslips were incubated with secondary antibodies at (1:200).
Nuclei staining was performed by exposing to 4, 6diamidino-2-phenylindole (1:2000) (MilliporeSigma) for $20 \mathrm{~s}$. The stained cells were visualized using an upright fluorescence microscope (Olympus, Tokyo, Japan).

\section{Xenograft model and optical imaging}

All animal procedures were conducted according to the guidelines of the Institutional Animal Care and Use Committee (IACUC No. KU17008) of Konkuk University. MDA-MB-231-EV and MDA-MB-231-IL-32 $\theta$ cells $\left(5 \times 10^{6}\right.$ cells $)$ were subcutaneously injected with Matrigel into the flanks of 5-week-old female athymic BALB/c nude mice (Nara Bio, Seoul, Korea). After 35 days, the tumors were harvested from euthanized mice. The tumor tissues were fixed with $10 \%$ formalin buffer, embedded in paraffin, and sectioned in $3 \mu \mathrm{m}$ thickness for use in immunohistochemical analyses. Tumor volume was calculated using the formula $\mathrm{V}\left(\mathrm{mm}^{3}\right)=$ (shortest $\operatorname{side}^{2} \times$ longest side) $/ 2$. For pre-and intra-operative tumor localization in the real-time resection, we conducted an in vivo tumor localization assay using the IRDye-800CW 2-DG (2-deoxy-D-glucose) optical probe (LI-COR Biosciences, Lincoln, NE). Tumor localization was detected using optical imaging, particularly in the near-infrared fluorescence range. The tumorigenicity of MDA-MB-231-EV and MDA-MB-231-IL-32 $\theta$ cells was assayed by intravenous injection of $1 \times 10^{6}$ cells resuspended in PBS into nude mice ( $n=5$ per group).

\section{Immunohistochemistry}

Formalin-fixed, paraffin embedded tumor tissue sections from mice were immersed in citrate buffer and boiled for $4 \mathrm{~min}$ in a microwave to retrieve antigens. Endogenous peroxidase activity was blocked with $3 \%$ (v/v) $\mathrm{H}_{2} \mathrm{O}_{2}$ for $10 \mathrm{~min}$. Non-specific binding sites were blocked with $1 \%$ BSA for $30 \mathrm{~min}$. Sections were incubated with the appropriate primary antibodies at $4{ }^{\circ} \mathrm{C}$ overnight and then the appropriate secondary antibodies for $1 \mathrm{~h}$ at RT. Diaminobenzidine tetrahydrochloride (Vector Laboratories, Burlingame, CA) was used as a substrate, and the sections were then counterstained with hematoxylin (MilliporeSigma).

\section{Statistical analysis}

Chi-square or Fisher's exact test was used to evaluate the relationship between IL-32 $\theta$ expression and clinicopathological status. The mRNA expression in tumor tissues and protein secretion in breast cancer patients' sera were analyzed by Mann-Whitney U test. Student's $t$-test were used to compare the two groups in in vitro and in vivo experiments. Statistical analyses were performed using GraphPad Prism software version 5.0. All $p$-values were two-sided, and $p<0.05$ was interpreted as being statistically significant. 


\section{Results}

\section{Association between tumor IL-32 $\theta$ mRNA levels and} breast tumor characteristics

To investigate whether IL-32 $\theta$ was expressed in the tissues of breast cancer patients, we performed RT-PCR analyses using our specific primers as described [17]. Of the total of 90 breast tumors examined, 35 tumors expressed IL-32 $\theta$. The clinicopathological features and IL-32 $\theta$ expression profiles for all patients are summarized in Table 1 . IL-32 $\theta$ expression was associated with tumor status, estrogen receptor (ER), progesterone receptor (PR), human epidermal growth factor receptor 2 (HER-2) status, and molecular classification characteristics. Interestingly, IL-32 $\theta$ appeared frequently in ER negative, PR negative, HER- 2 negative patients, and in those with triple negative-related breast cancer types (basal-like). Due to the lack of number of breast cancer patients with high metastasis status, the relationship between IL-32 $\theta$ and this status could not be assessed accurately. In general, IL-32 $\theta$ seemed to express in early tumor stage and be related triple negative breast cancer types.

\section{Opposing expression patterns of IL-32 $\theta$ and CCL18 in breast tumor tissues}

Among the factors secreted by macrophages, CCL18 was reported to have strong effects on breast cancer progression whereas macrophage-secreted IL- $1 \beta$, TNF- $\alpha$, and CCL5 were previously suppressed by IL-32 $\theta[12,18$, 22, 23]; thus, mRNA expression levels of these factors were measured. To identify the relationship between IL$32 \theta$ and breast cancer under the effect of TAMs, we

Table 1 Association of IL-32 $\theta$ mRNA expression and clinical characteristics of breast cancer patients

\begin{tabular}{|c|c|c|c|c|}
\hline \multirow[t]{3}{*}{ Characteristic } & \multirow{3}{*}{$\begin{array}{l}\text { Total } \\
n=90\end{array}$} & \multicolumn{2}{|c|}{ IL-32 $\theta$ expression } & \multirow[t]{3}{*}{$P$-value } \\
\hline & & \multirow{2}{*}{$\begin{array}{l}\text { Positive } \\
n(\%) \\
n=35\end{array}$} & \multirow{2}{*}{$\begin{array}{l}\text { Negative } \\
n(\%) \\
n=55\end{array}$} & \\
\hline & & & & \\
\hline \multicolumn{5}{|l|}{$\overline{\text { Age }}$} \\
\hline$>60$ & 9 & $4(44.4)$ & $5(55.6)$ & \multirow[t]{2}{*}{$0.7553^{b}$} \\
\hline$\leq 60$ & 81 & $31(38.3)$ & $50(61.7)$ & \\
\hline \multicolumn{5}{|l|}{ Tumor status } \\
\hline T0-1 & 29 & $16(55.1)$ & $13(44.8)$ & \multirow[t]{2}{*}{$0.0361^{a}$} \\
\hline $\mathrm{T} 2-3$ & 61 & $19(31.1)$ & $42(68.9)$ & \\
\hline \multicolumn{5}{|l|}{ Nodal status } \\
\hline NO-1 & 74 & $28(37.8)$ & $46(62.2)$ & \multirow[t]{2}{*}{$0.8036^{\mathrm{a}}$} \\
\hline N2-3 & 16 & $7(43.8)$ & $9(56.3)$ & \\
\hline \multicolumn{5}{|l|}{ Metastasis status } \\
\hline Yes & 3 & $0(0)$ & $3(100)$ & \multirow[t]{2}{*}{$0.1674^{b}$} \\
\hline No & 87 & $35(40.2)$ & $52(59.8)$ & \\
\hline \multicolumn{5}{|l|}{ Estrogen receptor (ER) } \\
\hline Positive & 64 & $18(28.1)$ & $46(71.9)$ & \multirow[t]{2}{*}{$0.001^{\mathrm{a}}$} \\
\hline Negative & 26 & $17(65.4)$ & $9(34.6)$ & \\
\hline \multicolumn{5}{|l|}{ Progesterone receptor (PR) } \\
\hline Positive & 53 & $14(26.4)$ & 39 (73.6) & \multirow[t]{2}{*}{$0.0037^{\mathrm{a}}$} \\
\hline Negative & 37 & $21(56.8)$ & $16(43.2)$ & \\
\hline \multicolumn{5}{|l|}{ Human epidermal growth factor receptor 2 (HER-2) } \\
\hline Positive & 56 & $17(30.4)$ & $39(69.6)$ & \multirow[t]{2}{*}{$0.0331^{\mathrm{a}}$} \\
\hline Negative & 34 & $18(52.9)$ & $16(47.1)$ & \\
\hline \multicolumn{5}{|l|}{ Molecular classification } \\
\hline Luminal A (ER+ PR+/- HER-2- Ki67 low) & 20 & $9(45)$ & $11(55)$ & \multirow[t]{4}{*}{$0.04^{a}$} \\
\hline Luminal B (ER+ PR+/- HER-2+/Ki67 high) & 44 & $11(25)$ & $33(75)$ & \\
\hline Basal-like (ER- PR- HER-2- EGFR+/Ki67 high) & 14 & $9(64.3)$ & $5(35.7)$ & \\
\hline HER2-enriched (ER-PR-HER-2+ Ki67 high) & 12 & $6(50)$ & $6(50)$ & \\
\hline
\end{tabular}


divided the breast tumor tissues in two groups according to CD206 expression (an M2 macrophage marker), with a CD206 $6^{+}$status $(n=33)$ and $\mathrm{CD}^{206^{-}}$tissues $(n=57)$ and measured CCL18, IL-1 $\beta$, TNF- $\alpha$, and CCL 5 mRNA by RT-qPCR (Fig. 1a). The results showed that CCL18 mRNA expression was significantly higher in in $\mathrm{CD}_{206}{ }^{+}$ group compared to $\mathrm{CD}^{2} 26^{-}$group in opposition to IL-32 $\theta$ expression $(p<0.05)$, whereas IL- $1 \beta$, TNF- $\alpha$, and CCL5 showed no difference between two groups (Fig. 1a). To clarify this relationship, the IL- $32 \theta^{+}$patient group $(n=35)$ and IL-32 $\theta^{-}$patient group $(n=55)$ were further assessed (Fig. 1b). Additionally, of the 55 serum samples collected

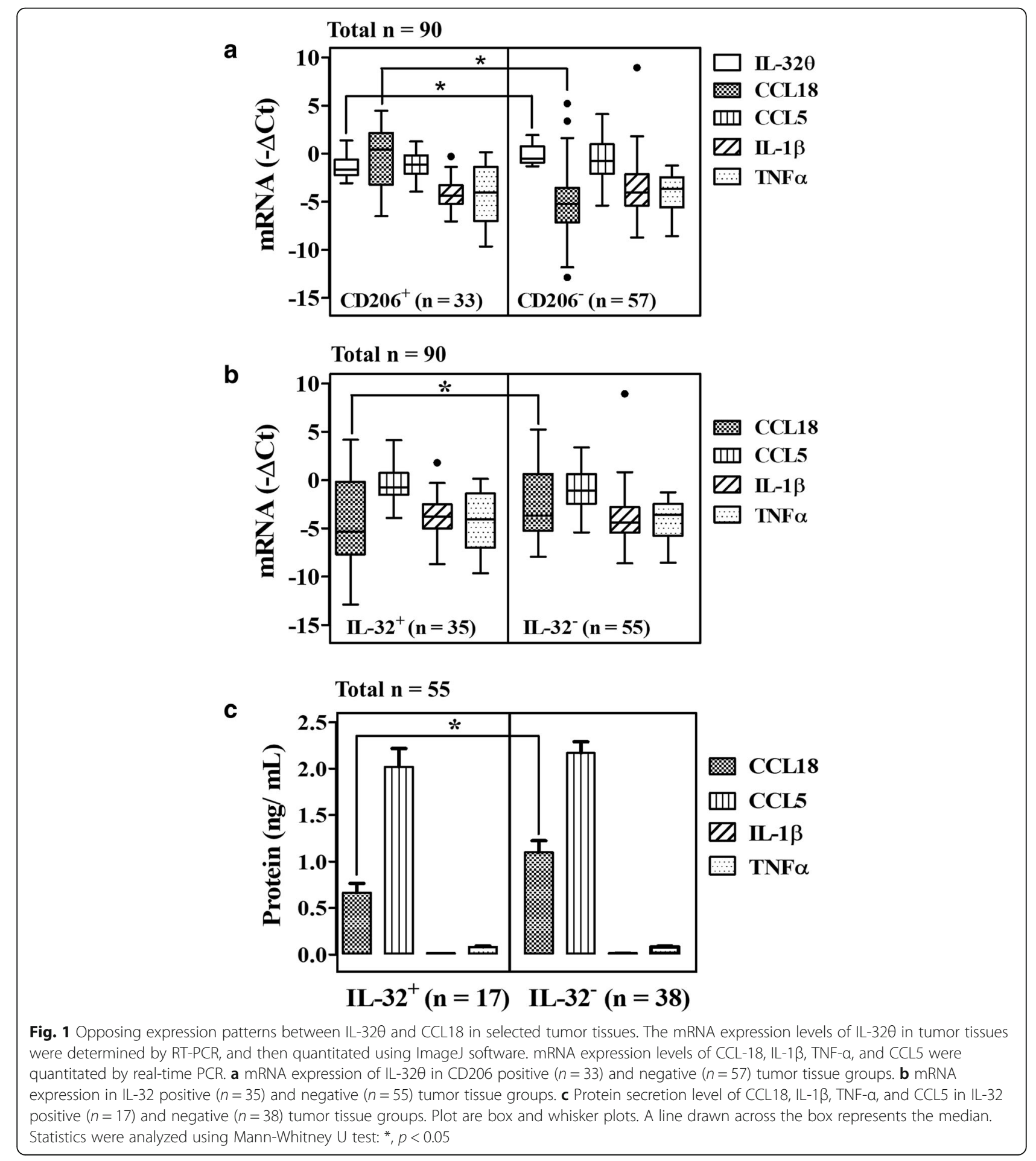


from breast cancer patients, protein secretion was measured in two groups IL- $32 \theta^{+}$patients $(n=17)$ and IL- $32 \theta^{-}$patients $(n=38)$ (Fig. 1c). Results indicated that in the presence of IL-32 $\theta$, CCL18 expression levels were lower than those without IL-32 $\theta$ while IL-1 $\beta$, TNF- $\alpha$, and CCL5 levels showed no difference between two groups. Unfortunately, secreted IL-1 $\beta$ and TNF- $\alpha$ were detected at very low level in the sera (Fig. 1c). These findings suggest that higher IL-32 $\theta$ expression in tumor tissue is accompanied by lower accumulation of CCL18 expression and vice versa while IL- $1 \beta$ or TNF- $\alpha$ or CCL5 expression are not affected by IL-32 $\theta$.

IL-32 $\theta$ reduces macrophage-regulated EMT, invasion, and migration in breast cancer cells in vitro

MDA-MB-231, a triple negative breast cancer cell line, has mesenchymal-like phenotype and can undergo EMT to be more aggressive during tumor progression [24]; thus, we generated an MDA-MB-231 cell line stably expressing IL-32 $\theta$ to study the function of IL-32 $\theta$ in EMT. Due to the endogenous IL-32 $\beta$ expression in MDA-MB231 cells [25], we used a specific primer set to recognize IL-32 $\beta$ and IL-32 $\theta$ as described previously [17]. The IL$32 \theta$ PCR product appeared at $299 \mathrm{bp}$, whereas the other isoforms appeared at $360 \mathrm{bp}$ because the IL-32 $\theta$ sequence does not include exon 6 , which is contained in IL-32 $\beta$ (Fig. 2a). PMA-treated THP-1 macrophages were reported to show equivalent properties to M2 macrophages [26], and this similarity was confirmed in the present study (Additional file 1: Figure S1a-e). Based on this phenomenon, CM from PMA-treated THP-1 macrophages was used to stimulate MDA-MB-231 progression (Fig. 2b). The cellular morphology of MDAMB-231 stably expressing IL-32 $\theta$ was observed without any stimulation, showing a more epithelial-like phenotype (Fig. 2c. upper panel). Consistent with previous research [12], MDA-MB-231 cells under CM treatment showed a more elongated shape and a mesenchymal-like phenotype compared to those in the non-treatment condition; however, IL-32 $\theta$ still moderated the morphological change to a more epithelial-like state (Fig. 2c. lower panel). Hence, it is assumed that IL-32 $\theta$ could potentially disrupt breast cancer EMT, invasion, and migration. For this reason, we evaluated whether IL-32 $\theta$ could regulate the epithelial marker, E-cadherin, and other tumor-promoting factors, COX-2 and MMP-9, stimulated by macrophages. As a result, the expression of E-cadherin under the stimulation of CM was downregulated in MDA-MB-231 EV cells as expected when MDA-MB-231 EV cells underwent EMT to become more aggressive, whereas it was significantly upregulated in MDA-MB-231-IL-32 $\theta$ cells (Fig. 2d-e). Significant downregulation of COX-2 and MMP-9 expression at the mRNA (Fig. 2d) and protein levels (Fig. 2e) were observed in MDA-MB-231-IL-32 $\theta$ cells as compared to MDA-MB-231 EV cells with or without CM stimulation. A decreasing pattern was also observed regarding MMP9 enzyme activity visualized by zymography (Fig. 2f). Moreover, macrophage-derived CCL18 was reported to create a feedback loop between macrophage and breast cancer cells by stimulating breast cancer-derived GM-CSF [12]. In this study, the GM-CSF mRNA and secretion levels were found to be significantly upregulated in the presence of $\mathrm{CM}$, which was markedly inhibited by IL-32 $\theta$ (Fig. 2d, g). To further determine the effects of IL-32 $\theta$ on cancer progression features, a transwell migration assay and a Matrigel invasion assay were performed (Fig. 2h-i). In the presence of CM, the stimulated MDA-MB-231-EV cells displayed increased rates of migration and invasion, and these rates were significantly reduced in MDA-MB-231-IL-32 $\theta$ cells (Fig. 2j) . These data supported the role of IL-32 $\theta$ in suppressing macrophage-induced breast cancer progression.

\section{IL-32 $\theta$ directly interacts with PKC $\delta$ to subsequently inhibit NF-KB and STAT3 pathways in vitro}

To investigate the precise mechanism by which IL-32 $\theta$ might regulate breast cancer progression, the relationship between IL-32 $\theta$ and PKC $\delta$ in breast cancer cells was explored based on previous studies demonstrating their interaction $[16,19]$. An immunoprecipitation assay showed that IL-32 $\theta$ could interact with only PKC $\delta$ upon PMA activation or CM stimulation in MDA-MB-231 IL$32 \theta$ cells (Fig. 3a). Therefore, it was hypothesized that IL-32 $\theta$ interacted with PKC $\delta$ upon stimulation with THP-1 macrophage CM to subsequently modulate downstream pathways in breast cancer cells. Based on the transcription factors inhibited by IL-32 $\theta$ previously [17-19], NF-kB and STAT3 were assumed to be involved in the IL-32 $\theta$-mediated PKC $\delta$ signaling. The western blot results revealed that in both $\mathrm{CM}$ treatment and non-treatment condition, IL-32 $\theta$ elevated IкB $\alpha$ expression, and inhibited phosphorylation of $\mathrm{I} \kappa \mathrm{B} \alpha$ and STAT3 at tyrosine 705 (Fig. 3b). Next, the nuclear translocation levels of STAT3, p65 plus p50 (two subunits of NF- $\mathrm{B}$ ), which were increased remarkably in the treatment with CM, were downregulated by IL-32 $\theta$ (Fig. 3c). Further, immunofluorescence analysis was performed to determine the location of IL-32 $\theta$, p65, and STAT3 in the nucleus and cytoplasm of MDA-MB-231 cells. The results were consistent with the western blot data, revealing that the fluorescence accumulation of p65 and STAT3 in the nucleus after stimulation with the CM was strongly reduced in IL-32 $\theta$-expressing cells (Fig. 3d-e). To confirm that IL-32 $\theta$ regulated NF- $\mathrm{kB}$ and STAT3 through $\mathrm{PKC} \delta$, a pharmacological inhibition of $\mathrm{PKC} \delta$ signaling by rottlerin [27] was applied before macrophage CM treatment. It was indicated that the PKC $\delta$ 


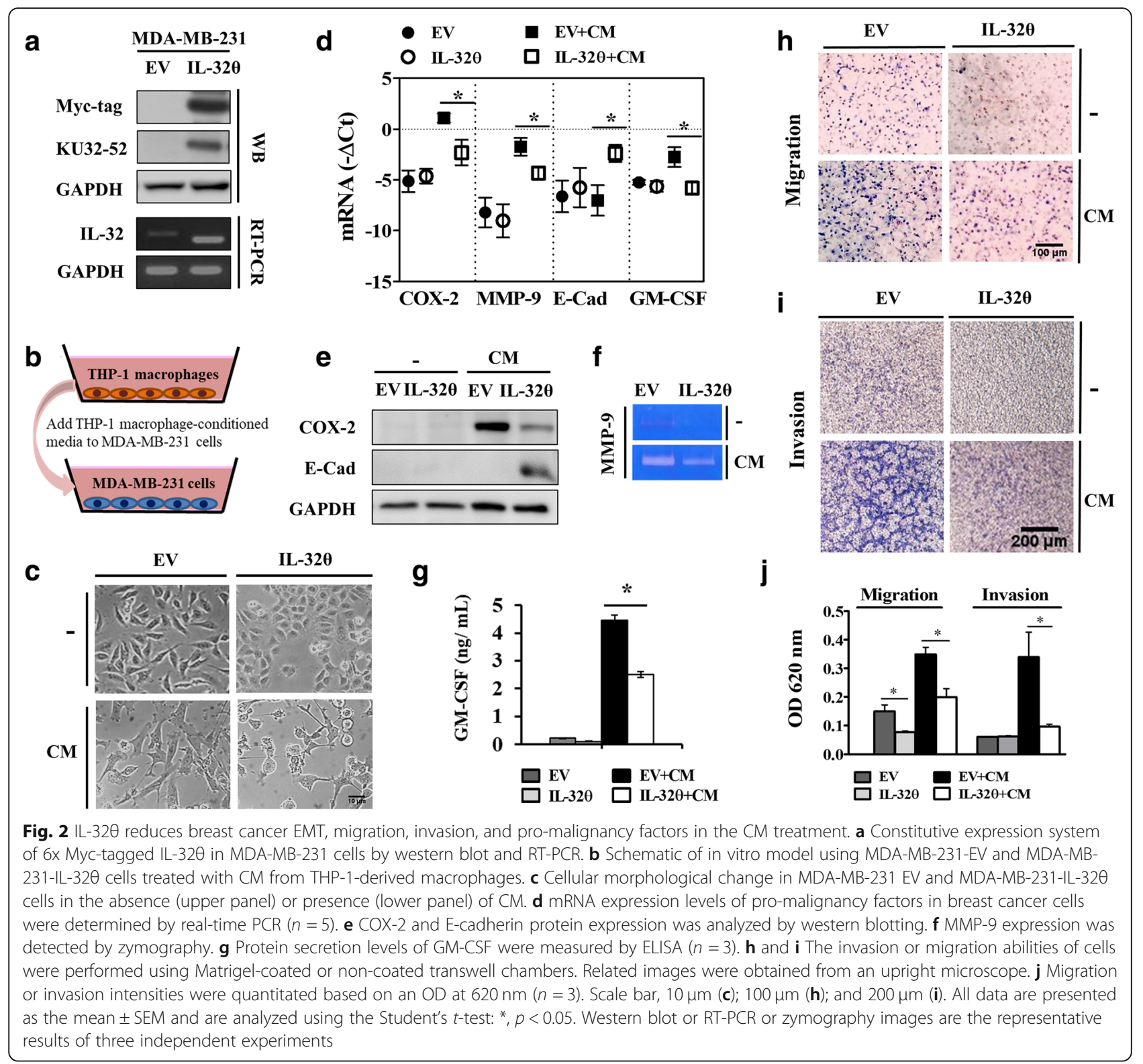

inhibitor could suppress ІкB $\alpha$ degradation and STAT3 phosphorylation, and additional effects of rottlerin and IL-32 $\theta$ on these signals were also observed (Fig. 3b). These data demonstrated that PKC $\delta$ mediated NF- $\mathrm{KB}$ and STAT3 signaling, and IL-32 $\theta$ inhibited these pathways.

\section{Blocking CCL18 signaling downregulates pro-malignancy factors and the PKC $\delta$ downstream pathway}

The secretion levels of CCL18 were significantly detected in the supernatant of THP-1-derived macrophage activated by PMA compared to the untreated control (Additional file 1: Figure S1f). Therefore, it was assumed that macrophage-secreted CCL18 might play regulating roles in EMT, invasion, and migration through PKC $\delta$ signaling which was suppressed by IL-32 $\theta$. To support this idea, CCL18 signaling was blocked using a neutralizing antibody in co-treatment with $\mathrm{CM}$ in both MDAMB-231 EV cells and MDA-MB-231 IL-32 $\theta$ cells. The disappearance of CCL18 signaling in MDA-MB-231 EV cells significantly downregulated the expression of COX-2, MMP-9, GM-CSF, and upregulated E-cadherin at both mRNA and protein levels (Fig. 4a-d). Moreover, the degradation of $\mathrm{I} \mathrm{KB} \alpha$, which represented the PKC $\delta$ downstream signaling, NF- $\mathrm{kB}$, was strongly reduced while the phosphorylated STAT3 at tyrosine 705 was slightly downregulated in the absence of CCL18 in MDA-MB-231 EV cells (Fig. 4b). Furthermore, the absence of CCL18 in CM impaired the 


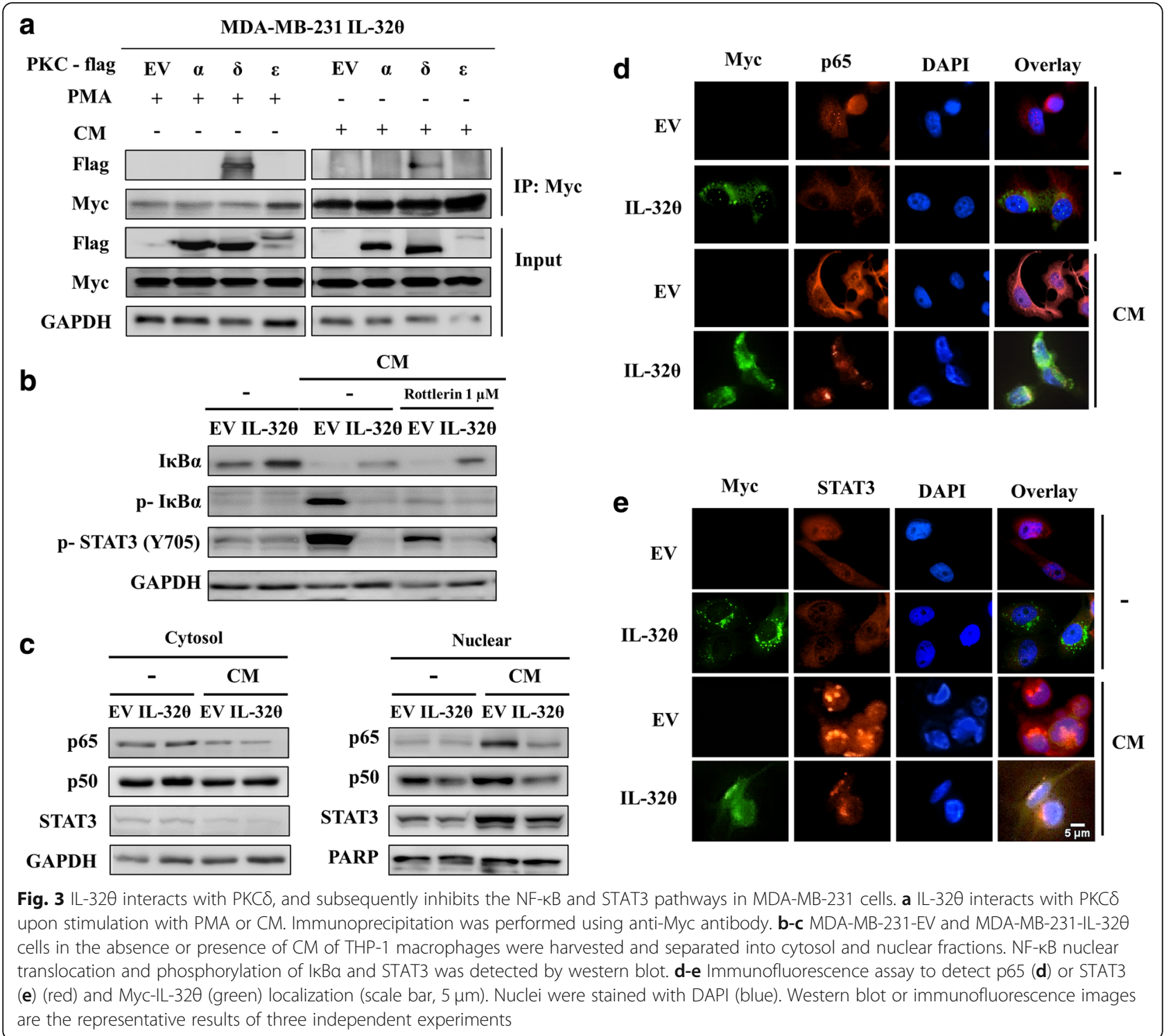

effects of CM on the migration and invasion rates of MDAMB-231 EV cells (Fig. 4e-f). These data suggest that CCL18 acts as an upstream activator of PKC $\delta$ signaling (including two downstream pathways, NF- $\mathrm{kB}$ and STAT3) to induce breast cancer progression. The cytokine CCL18 seemed to influence NF- $\mathrm{kB}$, and partly through STAT3 to stimulate E-cadherin, COX2, MMP-9, and GM-CSF expression. Notably, IL-32 $\theta$ might collaborate with neutralizing CCL18 antibody to display additive effects in the decrease of the expression of metastasis-related factors in the MDAMB-231 IL-32 $\theta$ cells as compared to that in the MDA-MB-231 EV cells (Fig. 4a-d). The results from migration and invasion assays, which were performed after blocking CCL18 signaling, indicated that the migration and invasion rates of the MDA-MB-231-IL-
$32 \theta$ cells were reduced to the minimum when compared to the IgG treated control group (Fig. 4 e-f). Therefore, it can be confirmed that CCL18 signaling is the main target of IL-32 $\theta$ to inhibit the macrophage-induced metastasis of breast cancer cells.

\section{IL-32 $\theta$ inhibits tumor formation of breast cancer cells in} vivo

To examine the cancerous properties of MDA-MB-231EV or MDA-MB-231-IL-32 $\theta$ cells, cells were injected to the flanks of mice in xenograft model (Fig. 5a). The immunohistochemistry results showed that the intensity of E-cadherin-positive cells was significantly increased, whereas the intensity for p65 and STAT3-positive cells were relatively decreased in the tumor tissue of the MDA-MB-231-IL-32 $\theta$ group compared to that of the 


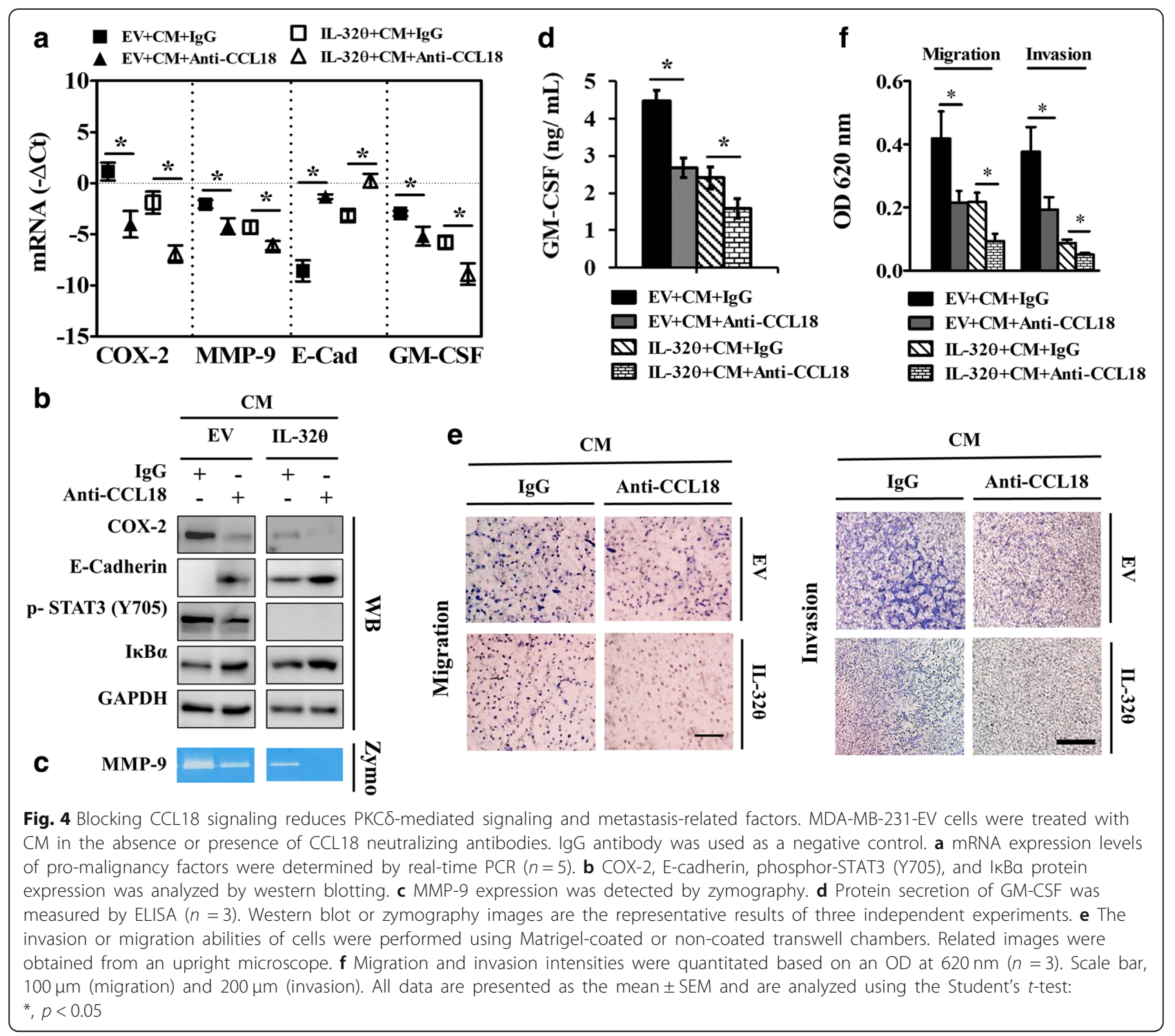

MDA-MB-231-EV group (Fig. 5b). Moreover, tumor volume was reduced significantly in the mouse group injected with MDA-MB-231-IL-32 $\theta$ cells (Fig. 5c). Furthermore, the mRNA levels of various tumor-promoting factors including COX-2, MMP-9, E-cadherin, and GMCSF were down-regulated in the MDA-MB-231-IL-32 $\theta$ group (Fig. 5d). These findings supported the idea that the antitumor activity of IL-32 $\theta$ was associated with the inactivation of NF- $\mathrm{kB}$ and STAT3 in tumor tissues. In another model, the MDA-MB-231-EV or MDA-MB231-IL-32 $\theta$ cells were treated with macrophage CM for $24 \mathrm{~h}$ before intravenous injection to nude mice (Fig. 5e). As shown in Fig. 5f-g, MDA-MB-231-EV cell tumors were large and emitted strong fluorescence signal, whereas a weaker pattern was observed in the mice treated with MDA-MB-231-IL-32 $\theta$ cells, suggesting potential effects of IL-32 $\theta$ on the tumor progression of stimulated breast cancer cells.

\section{Discussion}

Macrophages, a major component of the tumor microenvironment, can initiate and support the tumor progression and metastasis by secreting a range of growth factors, cytokines, and chemokines [28]. IL-32 was found to not only target cancer cells but might also target the tumor microenvironment [29]. Recent reports showed the correlation and different functions of IL-32 and its isoforms to various cancer diseases. As an example, IL$32 \gamma$ can inhibit colon cancer cell growth by targeting NF- $\mathrm{KB}$ and STAT3 pathways [30] while another isoform, IL-32 $\beta$, stimulates the migration of breast cancer cells through VEGF-STAT3 [25], and is involved in the 


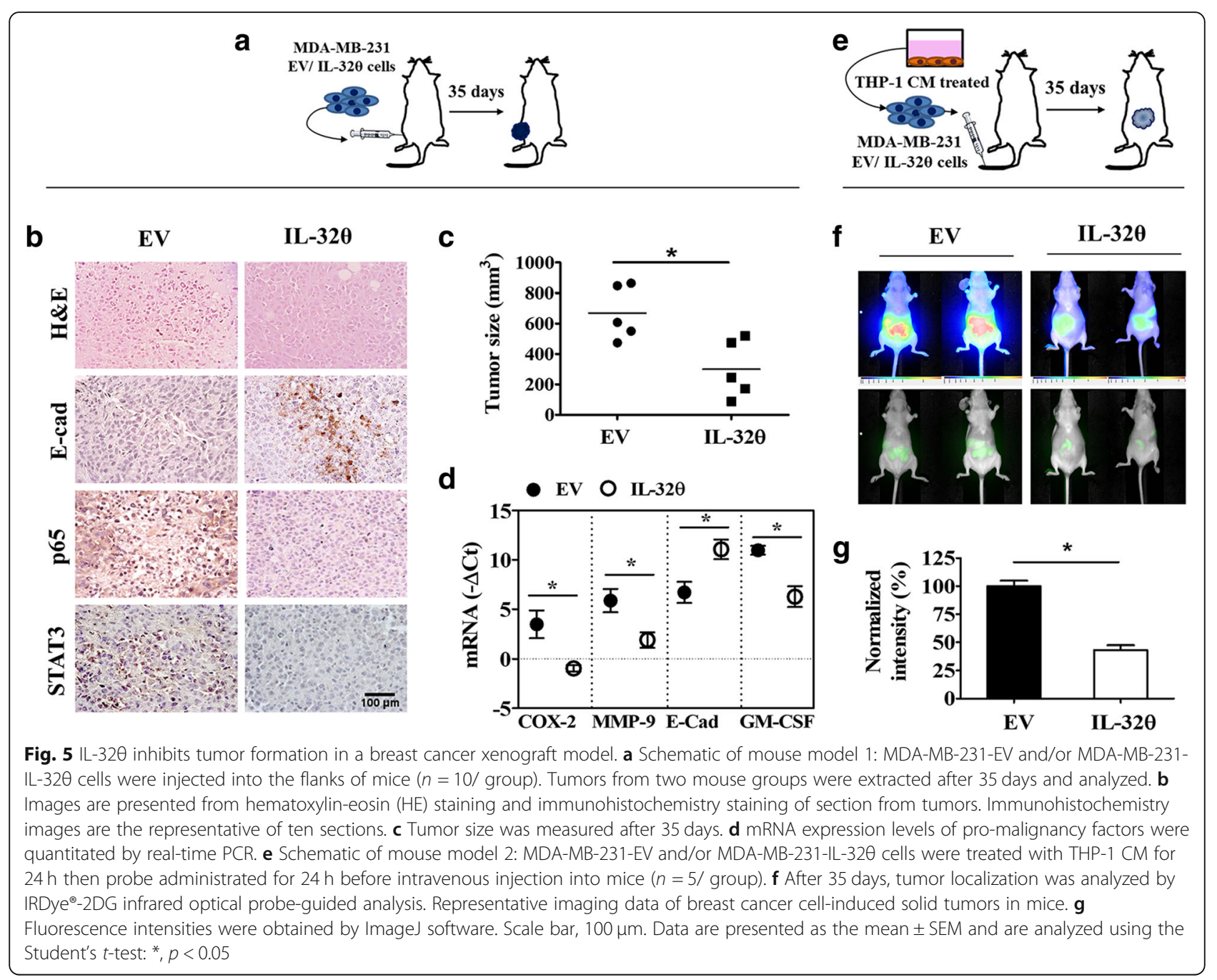

increase of glycolysis under hypoxic conditions which supports cancer cell growth [31]. Given these data, it appears that the effects of IL-32 on tumor development depend on both its isoforms and cancer types; however, the exact mechanisms remain unclear. Our previous data on IL-320, a recently discovered isoform, demonstrated its inhibition ability in macrophage differentiation [32], macrophage-secreted factors [16, 18, 19], and in colon cancer progression by regulating self-renewal and EMT [17]. In this study, three isoforms, IL-320, IL$32 \beta$, and IL-32 $\gamma$, were detected at different mRNA levels in 90 breast tumors. IL-32 $\beta$ exhibited the strongest expression which was compatible with its protumor effects reported in breast cancer [25] while IL-32 $\gamma$ was rarely expressed compared to the IL-32 $\theta$ isoform (Additional file 1: Fig. S2a-c). Given this, the current study attempted to discover the role of IL-32 $\theta$ in breast cancer progression and its tumor microenvironment. Our clinical data showed that IL-32 $\theta$ expression was associated with the negativity of ER, PR, and HER-2, and with triple negative related breast cancer types. Based on this point, we chose MDA-MB-231 cells, a highly aggressive, basal-like breast cancer cells with triple negative background [33], together with PMA-treated THP-1 macrophage cells to mimic the interaction between macrophages and cancer cells within the tumor microenvironment and evaluate the role of IL-32 $\theta$ on this interaction in vitro. This basal-like cell line is associated with both a poor prognosis and clinical outcome, due to its aggressiveness and high rate of metastasis [34]. We determined that the EMT phenotypic changes of MDA-MB-231 cells caused by stimulation of CM from THP-1 macrophages could be inhibited by IL-32 $\theta$. Moreover, invasion and migration rates were remarkably reduced in IL-32 $\theta$-expressing cells after $24 \mathrm{~h}$ treatment with $\mathrm{CM}$, suggesting that IL-32 $\theta$ could be a potential factor inhibiting macrophage-induced breast cancer progression. The 
interaction between macrophages and breast cancer cells has been reported to increase levels of various tumor promoting factors such as COX-2, and MMP-9 which, in turn, supports the breast malignancy and an increase of TAM density in the tumor microenvironment $[23,26,28,35]$. In agreement with these reports, the present study indicated that IL-32 $\theta$ downregulated COX-2, MMP-9, and E-cadherin expression in breast cancer cells stimulated by macrophages demonstrating a modulatory role of IL-32 $\theta$ in breast cancer development.

In addition, the precise mechanism by which IL-32 $\theta$ reduces the effects of macrophage on breast cancer progression was addressed based on previous studies detailing that IL-32 $\theta$ interacted directly with PKC $\delta$ to subsequently decrease STAT3 or NF- $к B$ signaling in PMA-activated THP-1 cells $[16,19]$. In line with this theory, the present study showed a direct interaction between IL-32 $\theta$ and PKC $\delta$ in breast cancer cells. Especially under CM treatment condition, IL-32 $\theta$ inhibited phosphorylation of IkB $\alpha$ plus STAT3, and nuclear translocation of NF- $\mathrm{kB}$ and STAT3 in MDAMB-231 cells (Fig. 3b). Moreover, interfering PKC $\delta$ signaling with rottlerin, a $\mathrm{PKC} \delta$ inhibitor, resulted in additive effects with IL-32 $\theta$ in the decrease of STAT3 phosphorylation and $I \kappa B \alpha$ degradation. Due to the fact that PKC $\delta$ mRNA expression was found to be significantly higher in ER-positive compared with ERnegative tumors [36], we applied this model on another breast cancer cell line with an estrogendependent background, MCF-7. However, IL-32 $\theta$ could not reduce any signal activated by macrophage $\mathrm{CM}$ in MCF-7 cells (Additional file 1: Figure S3a-b). Since MCF-7 represents epithelial-like cells and MDA-MB-231 represents mesenchymal-like cells, it is suggested that IL-32 $\theta$ seemed to effectively modulate the breast cancer with EMT-associated macrophages, which is essential for metastasis. The lack of PKC $\delta$ activation in MDA-MB-231 in the non-stimulated condition disappeared when MDA-MB-231 was cocultured with macrophage CM. Further studies are necessary to define the association between IL-32 $\theta$ and mesenchymal-like cells but not epithelial-like cells. In any case, these findings demonstrated that IL-32 $\theta$ targeted the interaction between macrophage and mesenchymal-like breast cancer, and there requires a specific macrophage-secreted factor to trigger PKC $\delta$ signaling in breast cancer which was inhibited by IL-32 $\theta$.

During the investigation of the IL-320-regulated signaling upstream factors, CCL18 was considered as a potential activator due to its presence in the THP-1 macrophage $\mathrm{CM}$, and the inverse expression between IL-32 $\theta$ and CCL18 in breast tumor tissues infiltrated with CD206+ macrophages. GM-CSF secreted from breast cancer cells activates macrophages to become CCL18-expressing TAM-like cells, which reciprocally supports GM-CSF secretion and furthers EMT of breast cancer cells [12]. Moreover, only GM-CSF significantly induced the production of TAM-related cytokines, and GM-CSF was found in CM from MDA-MB-231 cells but not MCF-7 cells [12]. Consistent with this study, our study found a decrease in the amount of GM-CSF secretion in IL-32 $\theta$-expressing MDA-MB-231 cells which might be stimulated by CCL18 from macrophages. Further, IL-32 $\theta$ did not suppress endogenous GM-CSF in MDA-MB-231 cells due to a lack of PKC $\delta$ activation and interaction. The transcription factors STAT3 and $\mathrm{NF}-\mathrm{kB}$ were also reported as the downstream factors regulated by PKC $\delta$ in cancer cells [37, 38]. In line with the idea that CCL18 is a stimulator of PKC $\delta$ signaling, our data demonstrated that blocking CCL18 signaling suppressed the expression of the PKC $\delta$ downstream factors STAT3 or NF- $\mathrm{kB}$ as well as various cancer-related factors. These results supported the idea that macrophage-secreted CCL18 might act as a stimulator of PKC $\delta$ signaling regulated by IL-32 $\theta$.

The present study also provides the first in vivo evidence of the suppressive function of IL- $32 \theta$ in breast cancer. A xenograft mouse model of MDA-MB-231-IL$32 \theta$ cells showed an increase of E-cadherin-positive cells, suggesting that IL-32 $\theta$ reversed the effects on EMT, whereas STAT3 and NF-kB-positive cells were much more abundant in the absence of IL-32 $\theta$. Another imaging model in which breast cancer cells were activated by macrophage CM to become more aggressive also supported that IL-32 $\theta$ could reduce the tumor localization clearly compared to the MDA-MB-231-EV group. These in vivo results are in accordance with the in vitro and clinical data demonstrating that IL-32 $\theta$ acts via PKC $\delta$ signaling to regulate the effects of macrophage-soluble factors on breast cancer cells.

The small population of patients' data collected recently does not allow us to perform a survival analysis to assess the relationship between IL-32 $\theta$ and the survival rate of breast cancer patients. Moreover, during studying about the effects of IL-32 $\theta$ on breast cancer cell proliferation, we have found that Bcl-2, an anti-apoptotic factor which has been proposed as a prognostic marker [39], was totally repressed by IL$32 \theta$ in vitro (data not shown). However, no significant change between two cell lines could be seen in the expression levels of the late apoptotic markers after $72 \mathrm{~h}$ from flow cytometry results (data not shown). Thus, it is necessary to study different types of cell death to understand by which mechanism IL-32 $\theta$ may affect the cell death. Finally, although there are some aspects described above to be considered, these will be the subjects of ongoing studies. 


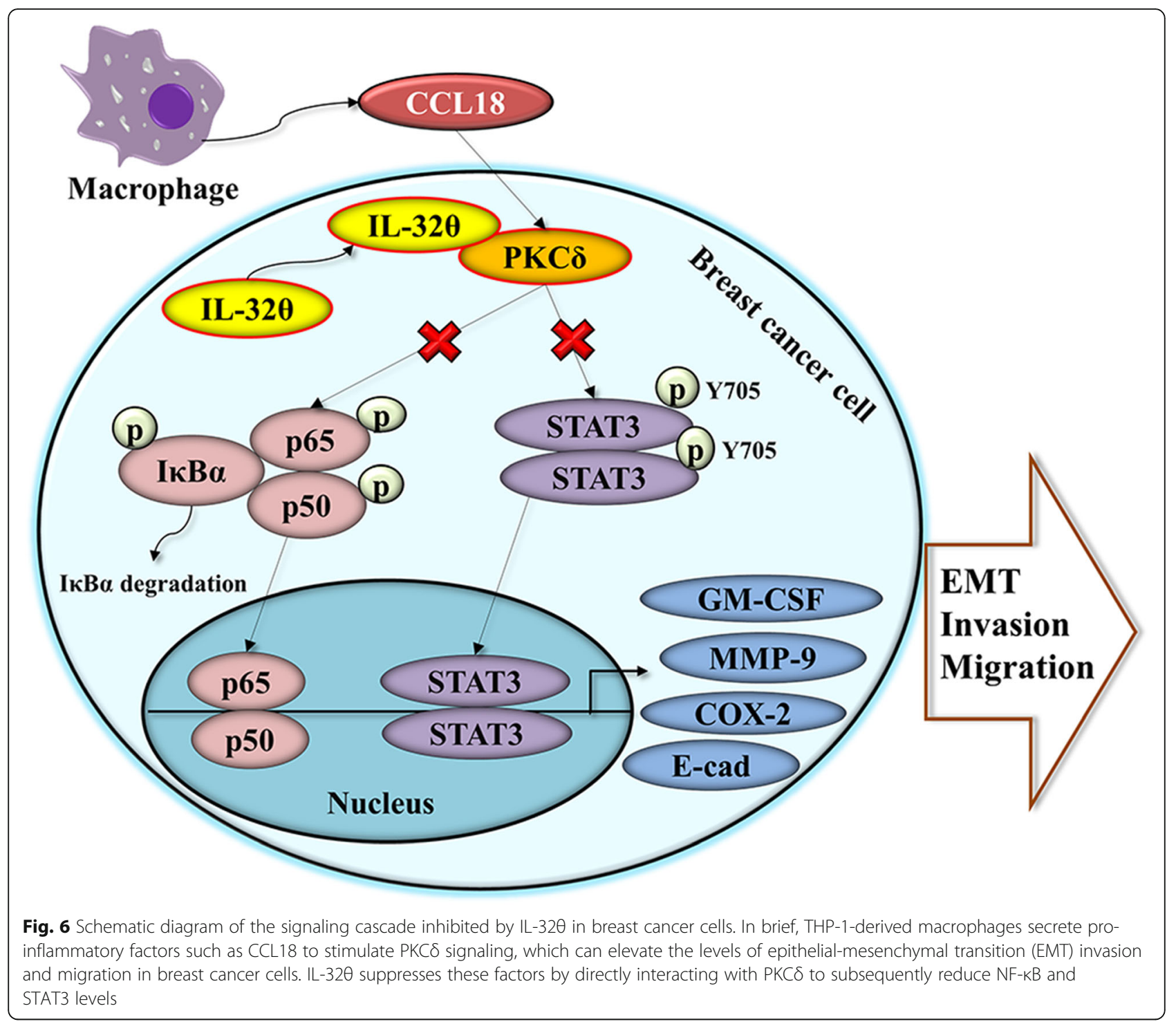

\section{Conclusions}

In conclusion, IL-32 $\theta$ inhibited EMT and metastasis in breast cancer cells by targeting CCL18 secreted from macrophages. A schematic diagram of this mechanism was shown in Fig. 6. The IL-320-mediated inhibition of macrophage-breast cancer cross-talk shows potential for a therapeutic strategy in blocking pro-metastatic activity of breast cancer.

\section{Additional files}

Additional file 1: Table S1. PCR primer sequences. Figure S1. PMAinduced macrophages show an M2-like phenotype. Figure S2. mRNA expression of IL-32 $\theta$, IL-32 3, IL-32 $\gamma$ in breast tumors. Figure S3. Effect of IL-32 $\theta$ on pro-malignancy and signaling factors in macrophage CMtreated MCF-7-EV and MCF-7-IL-320 cells. (PDF $514 \mathrm{~kb}$ )

Additional file 2: Raw data of Western blot. (PPTX $576 \mathrm{~kb}$ )

\section{Abbreviations}

CCL: Chemokine (C-C motif) ligand; CD206: Cluster of differentiation 206; CM: Conditioned media; COX-2: Cyclooxygenase 2; EMT: Epithelialmesenchymal transition; GM-CSF: Granulocyte-macrophage colonystimulating factor; IL: Interleukin; MMP-9: Matrix metallopeptidase 9; PKC: Protein kinase C; STAT3: Signal transducer and activator of transcription 3; TAMs: Tumor-associated macrophages

\section{Acknowledgements}

The biospecimens and characteristic data used for this study were provided by the Biobank of Chonnam National University Hwasun Hospital (Hwasun, Korea) and Korea University Guro Hospital (Seoul, Korea), members of the Korea Biobank Network.

\section{Authors' contributions}

TP and YB performed most of the experiments, analyzed the results and wrote the manuscript. TK conducted the optical imaging experiments. SK established the stable cell line. JO supported in vitro materials and contributed to edit the manuscript. JP and YC participated in xenograft experiments. DY and JTH supervised the study. All authors read and approved the final manuscript. 


\section{Author's information}

Not applicable.

\section{Funding}

This work was supported by a National Research Foundation of Korea (NRF) grant funded by the Korean Government (2018R1A2B2001225).

\section{Availability of data and materials}

All results of this study are presented in this article and additional files.

\section{Ethics approval and consent to participate}

The protocol using biospecimens and related data in this study was approved by the Institutional Review Board (7001355-201704-E-047) of Konkuk University, and all subjects provided informed consent. All animal procedures were conducted according to the guidelines and approved by the Institutional Animal Care and Use Committee (IACUC No. KU17008) of Konkuk University.

\section{Consent for publication}

Not applicable.

\section{Competing interests}

The authors declare that they have no competing interests.

\section{Author details}

'Department of Bioscience and Biotechnology, Konkuk University, 120 Neungdong-ro, Jayang-dong, Gwangjin-gu, Seoul 05029, Republic of Korea. ${ }^{2}$ Primate Resource Center, Division of Bioinfrastructure, Korea Research Institute of Bioscience and Biotechnology (KRIBB), Jeongeup, Jeollabuk-do, Republic of Korea. ${ }^{3}$ Department of Stem Cell and Regenerative Biotechnology, Konkuk University, Seoul, Republic of Korea. ${ }^{4}$ Department of Laboratory Animal Medicine, College of Veterinary Medicine, Konkuk University, Seoul, Republic of Korea. ${ }^{5}$ College of Pharmacy and Medical Research Center, Chungbuk National University, Osongsaengmyeong 1-ro, Osong-eup, Heungdeok-gu, Cheongju, Chungbuk 28160, Republic of Korea.

Received: 17 March 2019 Accepted: 20 May 2019

Published online: 24 May 2019

\section{References}

1. Bray F, Ferlay J, Soerjomataram I, Siegel RL, Torre LA, Jemal A. Global cancer statistics 2018: GLOBOCAN estimates of incidence and mortality worldwide for 36 cancers in 185 countries. CA Cancer J Clin. 2018:68:394-424.

2. Talmadge JE, Fidler IJ. AACR centennial series: the biology of cancer metastasis: historical perspective. Cancer Res. 2010;70:5649-69.

3. Steeg PS. Tumor metastasis: mechanistic insights and clinical challenges Nat Med. 2006;12:895-904.

4. Thiery JP. Epithelial-mesenchymal transitions in tumour progression. Nat Rev Cancer. 2002:2:442

5. Whiteside TL. The tumor microenvironment and its role in promoting tumor growth. Oncogene. 2008;27:5904-12.

6. Joyce JA, Pollard JW. Microenvironmental regulation of metastasis. Nat Rev Cancer. 2009:9:239-52.

7. Meads MB, Gatenby RA, Dalton WS. Environment-mediated drug resistance: a major contributor to minimal residual disease. Nat Rev Cancer. 2009;9: 665-74

8. Solinas G, Germano G, Mantovani A, Allavena P. Tumor-associated macrophages (TAM) as major players of the cancer-related inflammation. $J$ Leukoc Biol. 2009;86:1065-73.

9. Mahmoud SM, Paish EC, Powe DG, Macmillan RD, Grainge MJ, Lee AH, Ellis IO, Green AR. Tumor-infiltrating CD8+ lymphocytes predict clinical outcome in breast cancer. J Clin Oncol. 2011;29:1949-55.

10. Sica A, Mantovani A. Macrophage plasticity and polarization: in vivo veritas. J Clin Invest. 2012;122:787-95.

11. Biswas SK, Mantovani A. Macrophage plasticity and interaction with lymphocyte subsets: cancer as a paradigm. Nat Immunol. 2010;11:889-96.

12. Su S, Liu Q, Chen J, Chen J, Chen F, He C, Huang D, Wu W, Lin L, Huang W, et al. A positive feedback loop between mesenchymal-like cancer cells and macrophages is essential to breast cancer metastasis. Cancer Cell. 2014;25: $605-20$.
13. Kim SH, Han SY, Azam T, Yoon DY, Dinarello CA. Interleukin-32: a cytokine and inducer of TNF alpha. Immunity. 2005;22:131-42.

14. Kang JW, Park YS, Lee DH, Kim MS, Bak Y, Ham SY, Park SH, Kim H, Ahn JH, Hong JT, Yoon DY. Interaction network mapping among IL-32 isoforms. Biochimie. 2014;101:248-51.

15. Hong JT, Son DJ, Lee CK, Yoon DY, Lee DH, Park MH. Interleukin 32, inflammation and cancer. Pharmacol Ther. 2017;174:127-37.

16. Kim MS, Kang JW, Lee DH, Bak Y, Park YS, Song YS, Ham SY, Oh DK, Hong J, Yoon DY. IL-32theta negatively regulates IL-1 beta production through its interaction with PKCdelta and the inhibition of PU.1 phosphorylation. FEBS Lett. 2014;588:2822-9.

17. Bak Y, Kwon T, Bak IS, Hong J, Yu DY, Yoon DY. IL-32theta inhibits stemness and epithelial-mesenchymal transition of cancer stem cells via the STAT3 pathway in colon cancer. Oncotarget. 2016;7:7307-17.

18. Kim MS, Kang JW, Jeon JS, Kim JK, Kim JW, Hong J, Yoon DY. IL-32theta gene expression in acute myeloid leukemia suppresses TNF-alpha production. Oncotarget. 2015;6:40747-61.

19. Bak Y, Kang JW, Kim MS, Park YS, Kwon T, Kim S, Hong J, Yoon DY. IL32theta downregulates CCL5 expression through its interaction with PKCdelta and STAT3. Cell Signal. 2014;26:3007-15.

20. Kim KH, Shim JH, Seo EH, Cho MC, Kang JW, Kim SH, Yu DY, Song EY, Lee HG, Sohn JH, et al. Interleukin-32 monoclonal antibodies for immunohistochemistry, Western blotting, and ELISA. J Immunol Methods. 2008;333:38-50

21. Kim JH, Kim MS, Bak Y, Chung IM, Yoon DY. The cadin-2-en-1 beta-ol-1 betaD-glucuronopyranoside suppresses TPA-mediated matrix metalloproteinase9 expression through the ERK signaling pathway in MCF-7 human breast adenocarcinoma cells. J Pharmacol Sci. 2012:118:198-205.

22. Chen J, Yao Y, Gong C, Yu F, Su S, Chen J, Liu B, Deng H, Wang F, Lin L, et al. CCL18 from tumor-associated macrophages promotes breast cancer metastasis via PITPNM3. Cancer Cell. 2011;19:541-55.

23. Gan L, Qiu Z, Huang J, Li Y, Huang H, Xiang T, Wan J, Hui T, Lin Y, Li H, Ren G. Cyclooxygenase- 2 in tumor-associated macrophages promotes metastatic potential of breast cancer cells through Akt pathway. Int J Biol Sci. 2016;12:1533-43

24. Lacroix M, Leclercq G. Relevance of breast cancer cell lines as models for breast tumours: an update. Breast Cancer Res Treat. 2004;83:249-89.

25. Park JS, Choi SY, Lee JH, Lee M, Nam ES, Jeong AL, Lee S, Han S, Lee MS, Lim JS, et al. Interleukin-32beta stimulates migration of MDA-MB-231 and MCF-7cells via the VEGF-STAT3 signaling pathway. Cell Oncol (Dordr). 2013; 36:493-503.

26. Tjiu JW, Chen JS, Shun CT, Lin SJ, Liao YH, Chu CY, Tsai TF, Chiu HC, Dai YS, Inoue $\mathrm{H}$, et al. Tumor-associated macrophage-induced invasion and angiogenesis of human basal cell carcinoma cells by cyclooxygenase-2 induction. J Invest Dermatol. 2009:129:1016-25.

27. Kontny E, Kurowska M, Szczepanska K, Maslinski W. Rottlerin, a PKC isozymeselective inhibitor, affects signaling events and cytokine production in human monocytes. J Leukoc Biol. 2000;67:249-58.

28. Pollard JW. Tumour-educated macrophages promote tumour progression and metastasis. Nat Rev Cancer. 2004;4:71

29. Yun HM, Oh JH, Shim JH, Ban JO, Park KR, Kim JH, Lee DH, Kang JW, Park $\mathrm{YH}, \mathrm{Yu} \mathrm{D}$, et al. Antitumor activity of IL-32beta through the activation of lymphocytes, and the inactivation of NF-kappaB and STAT3 signals. Cell Death Dis. 2013;4:e640

30. Oh JH, Cho MC, Kim JH, Lee SY, Kim HJ, Park ES, Ban JO, Kang JW, Lee DH, Shim $\mathrm{JH}$, et al. IL-32gamma inhibits cancer cell growth through inactivation of NF-kappaB and STAT3 signals. Oncogene. 2011;30:3345-59.

31. Park JS, Lee S, Jeong AL, Han S, Ka HI, Lim JS, Lee MS, Yoon DY, Lee JH, Yang Y. Hypoxia-induced IL-32beta increases glycolysis in breast cancer cells. Cancer Lett. 2015:356:800-8.

32. Kim MS, Kang JW, Park YS, Lee DH, Bak Y, Kwon T, Yoon DY. IL-32theta inhibits monocytic differentiation of leukemia cells by attenuating expression of transcription factor PU.1. Oncotarget. 2015;6:4394-405.

33. Holliday DL, Speirs V. Choosing the right cell line for breast cancer research. Breast Cancer Res. 2011;13:215

34. Livasy CA, Karaca G, Nanda R, Tretiakova MS, Olopade OI, Moore DT, Perou CM. Phenotypic evaluation of the basal-like subtype of invasive breast carcinoma. Mod Pathol. 2006:19:264-71.

35. Hou Z, Falcone DJ, Subbaramaiah K, Dannenberg AJ. Macrophages induce COX-2 expression in breast cancer cells: role of IL-1beta autoamplification. Carcinogenesis. 2011;32:695-702. 
36. McKiernan E, O'Brien K, Grebenchtchikov N, Geurts-Moespot A, Sieuwerts AM, Martens JW, Magdolen V, Evoy D, McDermott E, Crown J, et al. Protein kinase Cdelta expression in breast cancer as measured by real-time PCR, western blotting and ELISA. Br J Cancer. 2008:99:1644-50.

37. Jain N, Zhang T, Kee WH, Li W, Cao X. Protein kinase C delta associates with and phosphorylates Stat3 in an interleukin-6-dependent manner. J Biol Chem. 1999;274:24392-400.

38. Lu ZG, Liu H, Yamaguchi T, Miki Y, Yoshida K. Protein kinase Cdelta activates RelA/p65 and nuclear factor-kappaB signaling in response to tumor necrosis factor-alpha. Cancer Res. 2009;69:5927-35.

39. Dawson SJ, Makretsov N, Blows FM, Driver KE, Provenzano E, Le Quesne J, Baglietto L, Severi G, Giles GG, McLean CA, et al. BCL2 in breast cancer: a favourable prognostic marker across molecular subtypes and independent of adjuvant therapy received. Br J Cancer. 2010;103:668-75.

\section{Publisher's Note}

Springer Nature remains neutral with regard to jurisdictional claims in published maps and institutional affiliations.

Ready to submit your research? Choose BMC and benefit from:

- fast, convenient online submission

- thorough peer review by experienced researchers in your field

- rapid publication on acceptance

- support for research data, including large and complex data types

- gold Open Access which fosters wider collaboration and increased citations

- maximum visibility for your research: over $100 \mathrm{M}$ website views per year

At $\mathrm{BMC}$, research is always in progress.

Learn more biomedcentral.com/submissions 